\title{
MicroRNA-4649-3p inhibits cell proliferation by targeting protein tyrosine phosphatase SHP-1 in nasopharyngeal carcinoma cells
}

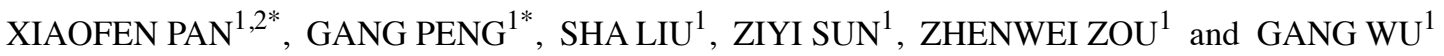 \\ ${ }^{1}$ Cancer Center, Union Hospital, Tongji Medical College, Huazhong University of Science and Technology, \\ Wuhan, Hubei 430022; ${ }^{2}$ Cancer Center, Affiliated Hospital of Guangdong Medical College, \\ Zhanjiang, Guangdong 524001, P.R. China
}

Received January 20, 2015; Accepted May 28, 2015

DOI: $10.3892 / \mathrm{ijmm} .2015 .2245$

\begin{abstract}
The present study aimed to investigate the influence of microRNA-4649-3p on nasopharyngeal carcinoma (NPC) cell proliferation and how it regulated SHP-1 expression. The online software TargetScan was used to predict the microRNAs targeting SHP-1 and identified that miR-4649-3p was one of the possible miRNAs targeting SHP-1. Subsequently, quantitative polymerase chain reaction (PCR) was used to detect the expression level of miR-4649-3p and SHP-1 mRNA in different NPC cell lines. The miR-4649-3p mimics and inhibitors were transfected into NPC cells and cell proliferation was examined by the MTT assay. The SHP-1 expression level was determined by PCR and western blot analysis. Lentivirus containing the SHP-1 gene and miR-4649-3p mimics was co-transfected into the NPC cells and cell proliferation was detected by the MTT assay. The expression level of miR-4649-3p and SHP-1 mRNA was negatively correlated in the NPC cell lines. miR-4649-3p mimics suppressed NPC cell proliferation whereas miR-4649-3p inhibitors promoted NPC cell proliferation. The SHP-1 expression level was suppressed when transfected with miR-4649-3p mimics in NPC cells. The miR-4649-3p inhibitors increased SHP-1 expression. The luciferase reporter assay showed that miR-4649-3p directly targeted SHP-1 by binding to the 3'-untranslated region of SHP-1 mRNA. Overexpression of SHP-1 inversed the inhibited effect of miR-4649-3p mimics on cell proliferation. In conclusion, miR-4649-3p inhibits cell proliferation by targeting SHP-1 in NPC cells.
\end{abstract}

Correspondence to: Dr Gang Wu, Cancer Center, Union Hospital, Tongji Medical College, Huazhong University of Science and Technology, 1277 Jiefang Dadao, Wuhan, Hubei 430022, P.R. China E-mail: xhzlwg@163.com

\section{${ }^{*}$ Contributed equally}

Abbreviations: NPC, nasopharyngeal carcinoma; RT, radiation therapy; IMRT, intensity-modulated radiotherapy; OS, overall survival; PFS, progression-free survival; UTR, untranslated region; SCR, control scrambled sequence

Key words: miRNA, nasopharyngeal carcinoma, protein tyrosine phosphatase, SHP-1, proliferation

\section{Introduction}

Nasopharyngeal carcinoma (NPC) is mainly prevalent in Southern China and Southeast Asia (1). Radiation therapy (RT) is the primary treatment for NPC. Intensity-modulated radiotherapy (IMRT), which exhibits fewer side effects compared with conventional two- and three-dimensional conformal radiation, is now widely used and achieves a good local control rate (2). However, for locally advanced or recurrent patients, treatment with IMRT alone often shows a poor prognosis (3). Besides RT, chemotherapy could also be administered as neoadjuvant, concurrent or adjuvant and is beneficial to NPC treatment. Even with the combination of RT and chemotherapy, local failure occurs in 7-13\% of patients following primary treatment for NPC (4). In addition to RT and the traditional chemotherapy, targeted therapy has attracted increasing attention for cancer treatment in the past few decades. Despite the success in lung (5), breast (6-8) and colorectal cancer $(9,10)$, phase II studies of targeted therapy showed insufficient results for NPC treatment. Drugs involved in the studies, including gefitinib, sorafenib and cetuximab, did not show a clear benefit to progression-free survival (PFS) or overall survival (OS) (11-14). These results suggested that traditional molecular targets, which are beneficial in other tumors, may not work well in NPC treatment. Therefore, it is necessary to discover new molecular targets in NPC treatment and improve the prognosis.

SHP-1 is an SH2 domain containing protein tyrosine phosphatase (PTP), which consists of 17 exons and 16 introns and spans $\sim 17$ kb DNA $(15,16)$. PTP SHP-1 expression increased in NPC tissue and was associated with radiation resistance and local recurrence (17). Knocking down SHP-1 by siRNA resulted in higher radiosensitivity in the NPC and lung cancer cell lines $(18,19)$. According to these studies, SHP-1 may be a potential target in NPC treatment and silencing SHP-1 may help improve the NPC prognosis.

The discovery of microRNAs (miRNAs or miRs) established a new era for targeted therapy. miRNAs are small non-coding RNAs that containing 19-25 nucleotides. These small non-coding RNAs regulate the target gene expression at the post-transcriptional level by binding to the 3 ' or 5'-untranslated region (UTR) and cause the degradation of target mRNA $(20,21)$. By regulating oncogene and anti-oncogene expression, miRNAs have important roles in tumor initiation 
Table I. Primer sequences for PCR.

\begin{tabular}{ll}
\hline Genes & \multicolumn{1}{c}{ Sequences } \\
\hline miR-4649-3p & Forward: 5'-ACACTCCAGCTGGGTCTGAGGCCTGCCTC-3' \\
& Reverse: 5'-CTCAACTGGTGTCGTGGAGTCGGCAATTCAGTTGAGTGGGGA-3' \\
U6 snRNA & Forward: 5'-CTTCGGCAGCACATATAC-3' \\
& Reverse: 5'-GGCCATGCTAATCTTCTC-3' \\
SHP-1 & Forward: 5'-ACCATCATCCACCTCAAGTACC-3' \\
& Reverse: 5'-CTGAGCACAGAAAGCACGAA-3' \\
$\beta$-actin & Forward: 5'-GATGAGATTGGCATGGCTTT-3' \\
& Reverse: 5'-CACCTTCACCGTTCCAGTTT-3'
\end{tabular}

and progression $(22,23)$. For that reason, miRNAs are closely associated with the tumor behaviors, as they can be conveniently detected in blood and other body fluid and they have great potential to become new biomarkers to monitor tumor development and therapeutic effect (24-26). As carcinogenesis is often correlated to abnormal miRNAs expression, reconstructing the normal miRNA network is a novel strategy in tumor treatment (27). A paradigm of miRNA therapeutics has been reported by Duchaine and Slacks (28). The paradigm pointed out a 'double strategies' concept: Using miRNAs as an anticancer agent or as cancer therapeutic targets (29).

In the present study, miRNA was used as an anticancer agent in NPC treatment. The miRNAs targeting SHP-1 were used to treat NPC cells, explore how it affects NPC cell proliferation and how it regulates SHP-1 expression.

\section{Materials and methods}

Cell culture. The NPC cell lines, CNE-1 and CNE-2, were purchased from the Cell Bank of Sun Yat-sen University (Guangzhou, China). 5-8F was obtained from the Cell Bank of Xiangya University (Changsha, China). C666-1, 6-10B, HNE-2, HNE-1 and HONE-1 were obtained from the Cell Bank of JRDUN Biotechnology (Shanghai, China). Cells were routinely cultured in RPMI-1640 (HyClone, Logan, UT, USA) supplemented with $12 \%$ fetal bovine serum (FBS; Gibco, Grand Island, NY, USA) and $1 \%$ penicillin/streptomycin (HyClone). The cells were maintained at $37^{\circ} \mathrm{C}$ in a humidified incubator with $5 \% \mathrm{CO}_{2}$.

Lentivirus, miRNA mimics or inhibitor transfection. miRNA mimics or inhibitors were purchased from Guangzhou RiboBio Co., Ltd. (Guangzhou, China). The control scrambled sequence (SCR) was used as a negative control. The miRNA mimics, inhibitors and SCR were transfected into NPC cells using Lipofectamine 2000 (Invitrogen, Carlsbad, CA, USA) according to the manufacturer's instructions. Lentiviruses containing the SHP-1 gene (LP-H1802-Lv201-C0010) and negative oligomers (LP-Neg-Lv201-0200) were purchased from GeneCopoeia (Guangzhou, China; 8.6×10 $10^{9}$ copies $/ \mathrm{ml}$ ). The two lentiviral stocks (50 $\mu \mathrm{l}$; LP-H1802Lv201 and LP-NegLv201) were transfected into the CNE-2 cells. Puromycin $(2 \mu \mathrm{g} / \mathrm{ml})$ was used to select the lentiviral-transfected cells. Total protein was isolated, and SHP-1 expression was detected by western blotting.
MTT assay. Cells were seeded into 96-well culture plates and incubated for 1, 2, 3, 4 and 5 days, respectively. The serum-containing culture medium was exchanged for normal culture medium not containing serum and $20 \mu \mathrm{l}$ MTT ( $5 \mathrm{mg} / \mathrm{ml}$; Sigma, St. Louis, MO, USA) was added. The samples were incubated in the dark for $4 \mathrm{~h}$. The culture medium was removed, $150 \mu \mathrm{l}$ dimethyl sulfoxide (Sigma) was added and the sample was slowly agitated for $15 \mathrm{~min}$. The optical density (OD) at 490/630 $\mathrm{nm}$ was tested using a microplate reader system (BioTek Instruments, Inc., Winooski, VT, USA).

Western blotting. Cells were harvested, lysed by the radioimmunoprecipitation assay (Google Biotechnology Ltd. Co., Wuhan, China) and total protein was extracted. Protein concentrations of the lysates were determined by the bicinchoninic acid protein assay system (Google Biotechnology Ltd. Co.). Equal amounts of protein were separated by $8-12 \%$ SDS-PAGE, and transferred to a polyvinylidene fluoride membrane (Millipore, Billerica, MA, USA). The membranes were blocked with 5\% bovine serum albumin and subsequently probed with either anti-SHP-1 (Cat. no. 1606-1; monoclonal, rabbit, targeted against mouse, rat, human; Epitomics, Burlingame, CA, USA) or anti-GAPDH (Cat. no. sc-25778; polyclonal, rabbit, targeted against human; Santa Cruz Biotechnology, Dallas, TX, USA) antibodies. Following washing, the membrane was incubated with the appropriate horseradish peroxidase secondary antibody (Cat. no. A24537; polyclonal, goat, targeted against rabbit; Life Technologies, Carlsbad, CA, USA) and visualized by chemiluminescence using a chemiluminescence kit (Invitrogen), and the specific bands were recorded by a UV transilluminator (Uvitec Ltd., Avebury House, Cambridge, UK). GAPDH protein levels were used as a control to verify equal protein loading.

Reverse transcription-quantitative polymerase chain reaction (RT-qPCR). Total RNA was extracted by TRIzol (Invitrogen) and reverse transcription was used to generate cDNA, according to the manufacturer's instructions of the Takara RT-PCR kit (Takara Bio, Shiga, Japan). Subsequently, qPCR was performed according to the manufacturer's instructions of SYBR-Green (Applied Biosystems, Foster City, CA, USA) in a PCR amplifier (ABI Prism 7000; Applied Biosystems). StepOne ${ }^{\mathrm{TM}}$ software v2.1 was used to analyze the data. Primer sequences are shown in Table I. 

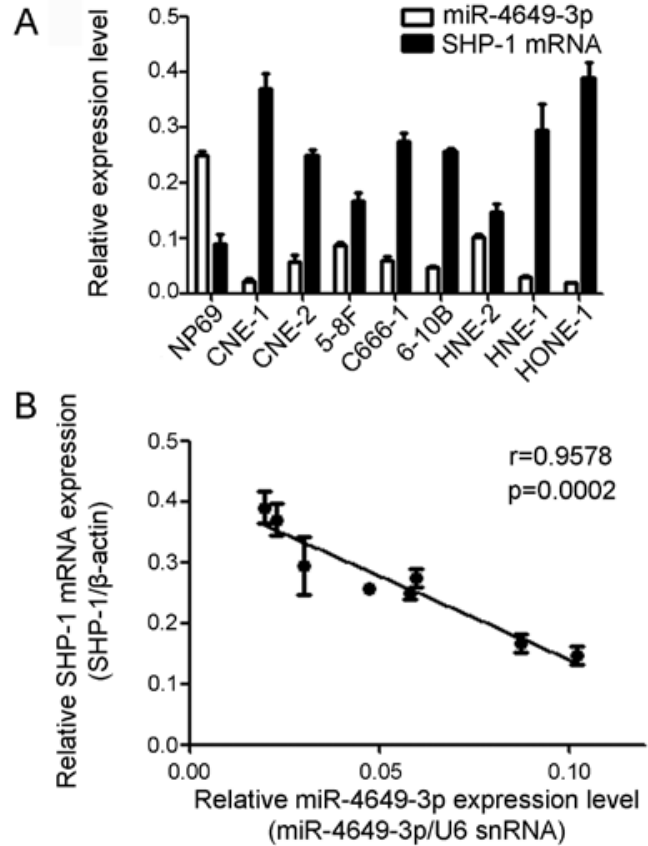

Figure 1. Expression of miR-4649-3p and SHP-1 mRNA in normal nasopharyngeal epithelia cell (NP69) and nasopharyngeal carcinoma (NPC) cells. (A) Compared with the normal nasopharyngeal epithelia cells, expression of miR-4649-3p in NPC cells was significantly decreased. However, SHP-1 mRNA in NPC cells was clearly increased. (B) Correlation between miR-4649-3p and SHP-1 mRNA was analyzed by the Pearson correlation test in NPC cells. The analysis results suggested a Pearson correlation parameter of $\mathrm{r}=-0.9578(\mathrm{P}=0.0002)$, meaning that miR-4649-3p expression was inversely correlated to SHP-1 expression. The data are presented as mean \pm standard deviation from at least three independent experiments.

Luciferase reporter assay. A 3'UTR fragment of SHP-1 containing the putative miR-4649-3p binding site (1018-1024 nucleotides) was amplified by PCR. The PCR product was subcloned into a p-GL3 vector (Promega, Madison, WI, USA) immediately downstream to the luciferase gene sequence. A p-GL3 construct containing the 3'UTR of SHP-1 with a mutant sequence of the miR-4649-3p binding site was also synthesized. All the constructs were verified by DNA sequencing. Cells were seeded in 96-well plates and subsequently co-transfected with $100 \mathrm{ng}$ constructs with or without miR-4649-3p mimics or inhibitors. At $48 \mathrm{~h}$ after transfection, luciferase activity was detected using a dual-luciferase reporter assay system (Promega) and normalized to Renilla activity.

Statistical analysis. Experimental data are expressed as the means \pm standard deviation from $\geq 3$ independent experiments. Differences in measured variables between experimental and control groups were assessed using the t-test (SPSS 21.0 software; IBM Corp., Armonk, NY, USA). P<0.05 was considered to indicate a statistically significant difference.

\section{Results}

miR-4649-3p is downregulated in NPC cell lines accompanied with SHP-1 upregulation. $\mathrm{qPCR}$ was used to detect the miR-4649-3p and SHP-1 mRNA expression level in the normal nasopharyngeal epithelia cell line and 8 different NPC cell lines. Compared with the normal nasopharyngeal epithelia
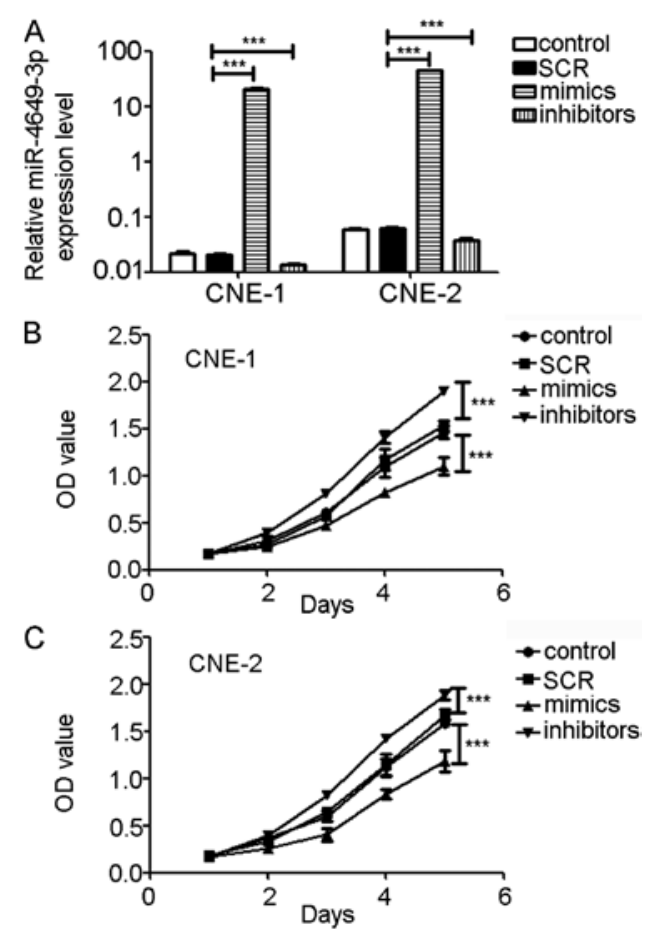

Figure 2. miR-4649-3p inhibited NPC cell proliferation. miR-4649-3p mimics and inhibitors were transfected into the nasopharyngeal carcinoma (NPC) cells CNE-1 and CNE-2. Subsequently, cell proliferation was determined by MTT assays. miR-4649-3p mimics increased miR-4649-3p expression by 900 - to 1,000-fold, while miR-4649-3p inhibitors decreased miR-4649-3p expression by $40-50 \%$. (A) miR-4649-3p expression level in CNE-1 and CNE-2. miR-4649-3p mimics inhibited NPC cell proliferation and miR-464p-3p inhibitors promoted proliferation. (B) Cell proliferation in CNE-1 presented by the optical density (OD) value. (C) Cell proliferation in CNE-2 presented by the OD value. The data are presented as mean \pm standard deviation from at least three independent experiments; ${ }^{* * *} \mathrm{P}<0.001$.

NP69 cells, the miR-4649-3p expression level was significantly decreased in the 8 NPC cell lines, CNE-1, CNE-2, HNE-1, HNE-2, 5-8F, 6-10B, C666-1 and HONE-1. However, the expression of SHP-1 mRNA was not the same as miR-4649-3p. The SHP-1 mRNA expression level in the NP69 cells was evidently lower compared to the NPC cells. The Pearson's correlation test was used to analyze the association between miR-4649-3p and SHP-1 mRNA expression in the 8 NPC cell lines. The results showed that miR-4649-3p expression was inversely correlated to the SHP-1 expression level. The Pearson correlation parameter was $\mathrm{r}=-0.9578(\mathrm{P}=0.0002)$ (Fig. 1).

miR-4649-3p inhibits NPC cell proliferation. To evaluate whether miR-4649-3p affects NPC cell proliferation, miR-4649-3p mimics and inhibitors were used to transfect CNE-1 and CNE-2 cells. SCR was used as a negative control. NPC cells transfected with miR-4649-3p inhibitors showed a 40-45\% decrease in miR-4649-3p expression. The miR-4649-3p mimic-transfected cells showed a 900- to 1,000-fold increase of miR-4649-3p expression. Three days after transfection, CNE-1 and CNE-2 cells were seeded in 96-wells plates and incubated for different times. Subsequently, cell proliferation, presented by the OD value, was determined. Cell proliferations were significantly inhibited in CNE-1 and CNE-2 cells treated with miR-4649-3p mimics. However, miR-4649-3p inhibitors significantly promoted cell proliferation (Fig. 2). 


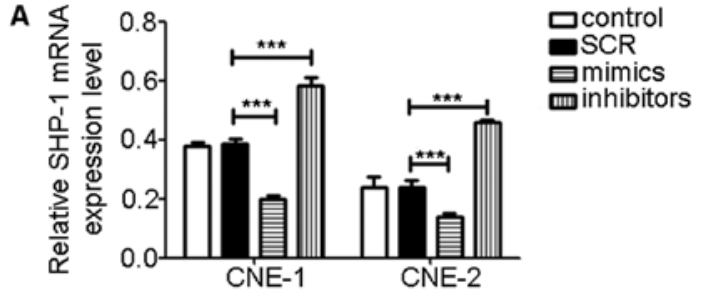

B

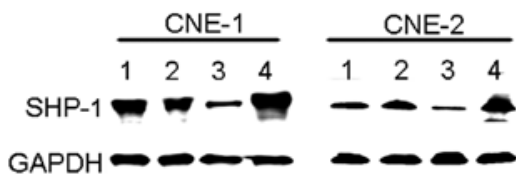

C

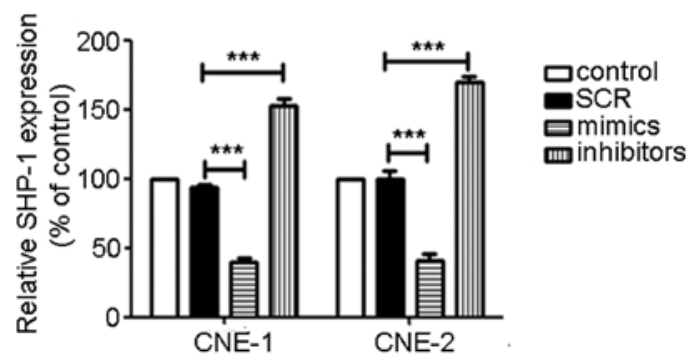

Figure 3. miR-4649-3p downregulates SHP-1 expression. miR-4649-3p mimics and inhibitors were transfected into nasopharyngeal carcinoma (NPC) cells CNE-1 and CNE-2. Subsequently, the SHP-1 expression level was determined by polymerase chain reaction and western blot analysis for the mRNA and protein levels, respectively. miR-4649-3p mimics significantly suppressed SHP-1 expression and miR-4649-3p inhibitors increased SHP-1 expression at the mRNA and protein levels. (A) SHP-1 mRNA expression level in CNE-1 and CNE-2. Data are presented as mean \pm standard deviation (SD) from three independent experiments; ${ }^{* * * *} \mathrm{P}<0.001$. (B) SHP-1 protein expression in CNE-1 and CNE-2. Lane 1, control; lane 2, control scrambled sequence (SCR); lane 3 , miR-46493p mimics; lane 4, miR-4649-3p inhibitors. Data are presented as one representative out of three independent experiments. (C) The optical density values were measured and presented as percentages of control. Data are displayed as mean $\pm \mathrm{SD}$ from three independent experiments; ${ }^{* * * *} \mathrm{P}<0.001$.

miR-4649-3p mimics downregulate SHP-1 expression. To investigate how miR-4649-3p affects SHP-1 expression, NPC cells were transfected with miR-4649-3p mimics and inhibitors. After three days, total RNA and protein were extracted and SHP-1 expression was examined by qPCR and western blot analysis. Between the control and SCR group, SHP-1 mRNA and protein expression did not have a significant difference. However, in the miR-4649-3p mimic-transfected group, SHP-1 expression was inhibited at the mRNA and protein level. In the miR-4649-3p inhibitor-transfected group, the SHP-1 expression level was evidently increased (Fig. 3).

miR-4649-3p directly targets SHP-1 3'UTR. To further understand how miR-4649-3p upregulated SHP-1 expression, luciferase reporter assays were performed. TargetScan human6.2 (www.targetscan.org) was used to predict the binding site of SHP-1 (PTPN6) with miR-4649-3p. According to the binding site sequence, a mutant sequence of the $3^{\prime} \mathrm{UTR}$ of SHP-1 was constructed by changing certain base pairs. Subsequently, the wild-type (wt) and mutant (mt) 3'UTR of SHP-1 were connected to a luciferase reporter gene and the wt-3'UTR and mt-3'UTR vectors were formed. The wt-3'UTR
A

wt SHP-1 3'UTR 5'-UCCUCAGGUGGCCAUGCCUCAGC-3'

miR-4649-3p 3'-ACCCCUCUCCGUCCGGAGUCU-5' mt SHP-1 3'UTR 5'-UCCUCAGGUGGCCAUGACCCAAC-3'

B

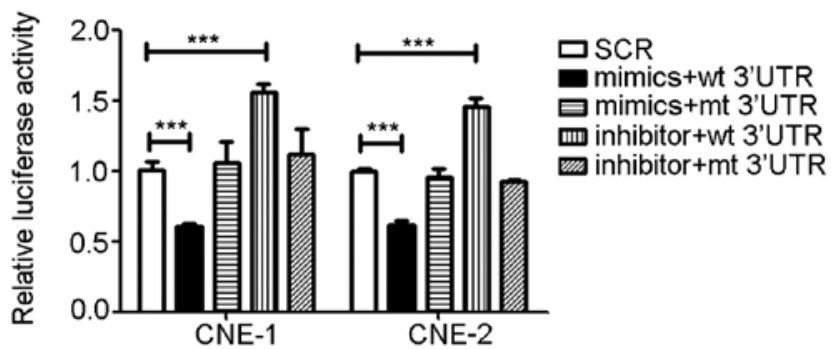

Figure 4. miR-4649-3p directly targets SHP-1 3'UTR. According to the binding site of SHP-1 3'UTR and miR-4649-3p, mt SHP-1 3'UTR was constructed by replacing certain base pairs. Subsequently, the luciferase reporter assay was used to examine the binding of miR-4649-3p and SHP-1 3'UTR in CNE-1 and CNE-2. When miR-4649-3p mimics and wt SHP-1 3'UTR were co-transfected into nasopharyngeal carcinoma (NPC) cells, luciferase activity was significantly inhibited. When co-transfected with miR-4649-3p inhibitors and wt SHP-1 3'UTR, luciferase activity was clearly increased. However, in the mt SHP-1 3'UTR groups, the effect of miR-4649-3p mimics and inhibitors was abolished. (A) Sequence of miR-4649-3p, wt SHP-1 3'UTR and mt SHP-1 3'UTR. (B) Luciferase activity of CNE-1 and CNE-2. Data are presented as mean \pm standard deviation from three independent experiments; ${ }^{* * *} \mathrm{P}<0.001$. SCR, control scrambled sequence; UTR, untranslated region; wt, wild-type; mt, mutant.

and miR-4649-3p mimics or inhibitors were co-transfected into the NPC cells. Luciferase activity of the miR-4649-3p mimics group was significantly suppressed while the activity of miR-4649-3p inhibitors groups was clearly increased. However, when the miR-4649-3p mimics or inhibitors was co-transfected with mt-3'UTR, the impact of the miR-4649-3p mimics or inhibitors on luciferase activity was abolished (Fig. 4).

Overexpression of SHP-1 abolishes the inhibition effect of $m i R-4649-3 p$. The lentivirus containing the SHP-1 gene (LP-H1802lv201) was used to transfect the NPC cells, CNE-1 and CNE-2, and SHP-1 overexpressed NPC cells were generated. LP-Neglv201 was a negative control. The MTT assay was performed again and an increasing SHP-1 expression level promoted cell proliferation. However, transfection of the miR-4649-3p mimics into NPC cells inhibited cell proliferation. When miR-4649-3p mimics and LP-H1802lv201 were co-transfected into NPC cells, the influence of SHP-1 on cell proliferation was abolished (Fig. 5)

\section{Discussion}

miRNAs have attracted increasing attention since being discovered, particularly in the field of cancer diagnosis and therapy. Since they can be detected in peripheral blood, body fluid and even in feces, they are extremely convenient for clinical use. Thus far, a significant number of studies have been performed utilizing miRNAs, from tumor markers to cancer therapy $(21,30-37,39)$. Traditional chemotherapeutic agents have certain limitations, among which severe side effects and drug resistance are the 
A

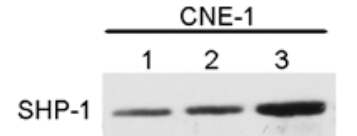

GAPDH

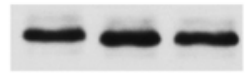

$\mathrm{B}$

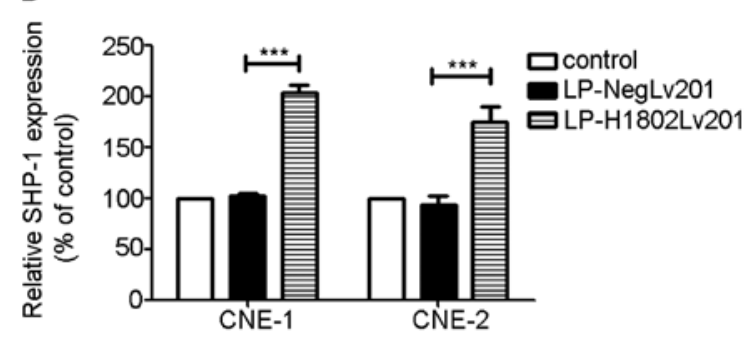

C $\quad \rightarrow$ LP-NegLV201+SCR
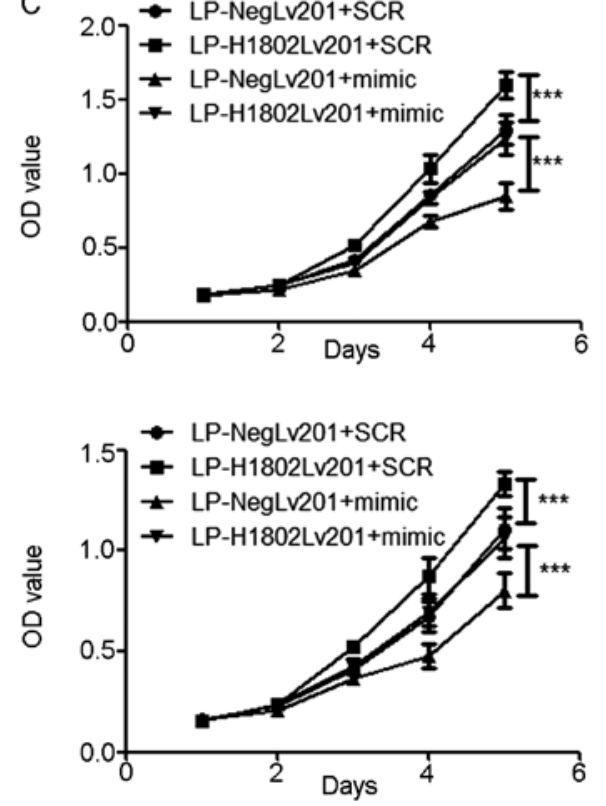

Figure 5. Overexpression of SHP-1 abolishes the inhibition effect of miR-4649-3p. Lentivirus containing the SHP-1 gene (LP-H1802lv201) was used to transfect nasopharyngeal carcinoma (NPC) cells and obtain SHP-1 overexpressed NPC cells. LP-Neglv201 was a negative control. Western blot analysis was used to detect the transfected efficiency. LP-H1802lv201 promoted NPC cell proliferation in contrast to LP-Neglv201. When LP-H1802lv201 and miR-4649-3p mimics were co-transfected into NPC cells, the influence of LP-H1802lv201 was revered. (A) SHP-1 expression level in CNE-1 and CNE-2. Lane 1, CNE-1/CNE-2; lane 2, LP-Neglv201; lane 3, LP-H1802lv201. (B) The optical density values were measured and presented as percentages of control. Data are presented as mean \pm standard deviation from three independent experiments; ${ }^{* * * *} \mathrm{P}<0.001$. (C) Cell proliferation of CNE-1 and CNE-2, represented by the optical density value.

major ones. Through binding to target gene mRNA, miRNAs can knockdown the oncogene expression. The strategy of using miRNAs to inhibit oncogene expression provides a new therapeutic approach with high selectivity and small side effects. Increasing research has focused on using miRNAs as new therapeutic agents in tumor therapy. Inhibiting NF- $\kappa \mathrm{B}$ activation by miR-31 in breast cancer promoted cell death and increased cell sensitivity to ionizing radiation (38). Zhang et al (39) found that miR-451 can increase radiosensitivity of NPC cells by targeting RAB14. Recently, certain investigators have tried to deliver miRNAs in combination with traditional chemotherapeutic drugs in cancer therapy. They found that the combination of miRNAs and chemotherapeutic drugs was effective and could enhance the sensitivity of cancer cells (40).

In the past few years, studies of miRNAs in NPC have achieved significant advancements. A number of miRNAs have been found to be associated with NPC formation, progression and other malignant behaviors. The majority of these miRNAs have roles in NPC pathology by modulating their target gene expression, including cell proliferation, migration, invasion and radiosensitivity.

SHP-1 is an SH2 domain containing PTP, which has 17 exons and 16 introns and spans $\sim 17 \mathrm{~kb}(15,16)$. It is strongly expressed in normal hematopoietic cells and weakly expressed in certain hematological malignancies. However, specific studies have found that SHP-1 is highly expressed in certain epithelial carcinoma cells, such as ovarian and breast cancer cell lines (41-45). It has been reported that SHP-1 was involved in NPC initiation and progression. Knocking down SHP-1 enhances radiosensitivity in the NPC and NSCLC cells (17-19). The present study aimed to find a way in which SHP-1 expression could be downregulated and thus inhibit NPC malignant behaviors. The achievements reached in miRNA research have offered a new insight.

As miRNAs can selectively regulate target gene expression at a post-transcription level, our attention was focused on miRNAs targeting SHP-1. First, the miRNAs that target SHP-1 were predicted using TargetScan human6.2. Ten candidate miRNAs were selected and tested. Among these, miR-4649-3p suppressed SHP-1 expression most significantly (data not shown). Thus, the present study aimed to investigate the influence of miR-4649-3p on NPC cell proliferation and SHP-1 expression. The miR-4649-3p and SHP-1 expression levels were examined in normal nasopharyngeal epithelia NP69 cells and 8 NPC cells. Compared with the normal nasopharyngeal epithelia cells, miR-4649-3p was downregulated whereas SHP-1 was upregulated in NPC cell lines. Increasing miR-4649-3p expression ectopically inhibited NPC cell proliferation. PCR and western blot analysis showed that miR-4649-3p mimics suppressed SHP-1 expression. The luciferase reporter assay indicated that miR-4649-3p suppressed SHP-1 expression by binding to SHP-1 3'UTR and inhibiting SHP-1 mRNA translation. To verify that miR-4649-3p inhibited NPC cell proliferation by targeting SHP-1, we ectopically expressed SHP-1 in NPC cells and found that the effect of the miR-4649-3p mimics was inversed. Therefore, ectopically expressing miR-4649-3p in NPC cells may potentially be a new strategy in NPC treatment.

\section{Acknowledgements}

The present study was supported by grants from the National Natural Sciences Foundation of China (no. 81301976) and the Natural Sciences Foundation of Hubei Province (no. 2012FFB02324).

\section{References}

1. Wee JT, Ha TC, Loong SL and Qian CN: Is nasopharyngeal cancer really a 'Cantonese cancer'? Chin J Cancer 29: 517-526, 2010.

2. Ng WT, Lee MC, Hung WM, Choi CW, Lee KC, Chan OS and Lee AW: Clinical outcomes and patterns of failure after intensity-modulated radiotherapy for nasopharyngeal carcinoma. Int J Radiat Oncol 79: 420-428, 2011. 
3. Tham IW, Hee SW, Yeo RM, Salleh PB, Lee J, Tan TW, Fong KW, Chua ET and Wee JT: Treatment of nasopharyngeal carcinoma using intensity-modulated radiotherapy-the national cancer centre singapore experience. Int J Radiat Oncol 75: 1481-1486, 2009.

4. Stoker SD, van Diessen JN, de Boer JP, Karakullukcu B Leemans CR and Tan IB: Current treatment options for local residual nasopharyngeal carcinoma. Curr Treat Options Oncol 14: 475-491, 2013.

5. Lee CK, Brown C, Gralla RJ, Hirsh V, Thongprasert S, Tsai CM, Tan EH, Ho JC, Chu da T and Zaatar A: Impact of EGFR inhibitor in non-small cell lung cancer on progression-free and overall survival: A meta-analysis. J Natl Cancer I 105: 595-605, 2013.

6. Dahabreh IJ, Linardou H, Siannis F, Fountzilas G and Murray S: Trastuzumab in the adjuvant treatment of early-stage breast cancer: A systematic review and meta-analysis of randomized controlled trials. Oncologist 13: 620-630, 2008.

7. Romond EH, Perez EA, Bryant J, Suman VJ, Geyer CE Jr, Davidson NE, Tan-Chiu E, Martino S, Paik S, Kaufman PA, et al: Trastuzumab plus adjuvant chemotherapy for operable HER2-positive breast cancer. N Engl J Med 353: 1673-1684, 2005.

8. Marty M, Cognetti F, Maraninchi D, Snyder R, Mauriac L, Tubiana-Hulin M, Chan S, Grimes D, Antón A, Lluch A et al: Randomized phase II trial of the efficacy and safety of trastuzumab combined with docetaxel in patients with human epidermal growth factor receptor 2-positive metastatic breas cancer administered as first-line treatment: The M77001 study group. J Clin Oncol 23: 4265-4274, 2005

9. Cheng AL, Li J, Vaid AK, Ma BB, Teh C, Ahn JB, Bello M, Charoentum C, Chen LT, de Lima Lopes G Jr, et al: Adaptation of international guidelines for metastatic colorectal cancer: An asian consensus. Clin Colorectal Cancer 13: 145-155, 2014.

10. Adachi T, Hinoi T, Egi H, Shimomura $M$ and Ohdan $H$ : Oxaliplatin and molecular-targeted drug therapies improved the overall survival in colorectal cancer patients with synchronous peritoneal carcinomatosis undergoing incomplete cytoreductive surgery. Surg Today: Aug 26, 2014 (Epub ahead of print).

11. Chua DT, Wei WI, Wong MP, Sham JS, Nicholls J and Au GK: Phase II study of gefitinib for the treatment of recurrent and metastatic nasopharyngeal carcinoma. Head Neck 30: 863-867, 2008.

12. Ma B, Hui EP, King A, To KF, Mo F, Leung SF, Kam M, Lo YM Zee B, Mok T, Ahuja A, et al: A phase II study of patients with metastatic or locoregionally recurrent nasopharyngeal carcinoma and evaluation of plasma Epstein-Barr virus DNA as a biomarker of efficacy. Cancer Chemother Pharmacol 62: 59-64, 2008.

13. Elser C, Siu LL, Winquist E, Agulnik M, Pond GR, Chin SF, Francis P, Cheiken R, Elting J, McNabola A, et al: Phase II trial of sorafenib in patients with recurrent or metastatic squamous cell carcinoma of the head and neck or nasopharyngeal carcinoma. J Clin Oncol 25: 3766-3773, 2007.

14. Chan AT, Hsu MM, Goh BC, Hui EP, Liu TW, Millward MJ, Hong RL, Whang-Peng J, Ma BB, To KF, et al: Multicenter, phase II study of cetuximab in combination with carboplatin in patients with recurrent or metastatic nasopharyngeal carcinoma. J Clin Oncol 23: 3568-3576, 2005

15. Banville D, Stocco R and Shen SH: Human protein tyrosine phosphatase 1C (PTPN6) gene structure: Alternate promoter usage and exon skipping generate multiple transcripts. Genomics 27 165-173, 1995 .

16. Evren S, Wan S, Ma XZ, Fahim S, Mody N, Sakac D, Jin T and Branch DR: Characterization of SHP-1 protein tyrosine phosphatase transcripts, protein isoforms and phosphatase activity in epithelial cancer cells. Genomics 102: 491-499, 2013.

17. Peng G, Cao R, Xue J, Li P, Zou Z, Huang J and Ding Q: Increased expression of SHP-1 is associated with local recurrence after radiotherapy in patients with nasopharyngeal carcinoma. Radiol Oncol 48: 40-49, 2014.

18. Peng G, Cao RB, Li YH, Zou ZW, Huang J and Ding Q: Alterations of cell cycle control proteins SHP-1/2, p16, CDK4 and cyclin D1 in radioresistant nasopharyngeal carcinoma cells. Mol Med Rep 10: 1709-1716, 2014

19. Cao R, Ding Q, Li P, Xue J, Zou Z, Huang J and Peng G: SHP1-mediated cell cycle redistribution inhibits radiosensitivity of non-small cell lung cancer. Radiat Oncol 8: 178, 2013.

20. Li G, Qiu Y, Su Z, Ren S, Liu C, Tian Y and Liu Y: Genome-wide analyses of radioresistance-associated miRNA expression profile in nasopharyngeal carcinoma using next generation deep sequencing. PloS One 8: e84486, 2013.

21. Li G, Liu Y, Su Z, Ren S, Zhu G, Tian Y and Qiu Y: MicroRNA-324-3p regulates nasopharyngeal carcinoma radioresistance by directly targeting WNT2B. Eur J Cancer 49: 2596-2607, 2013.

22. Zhang H, Pu J, Qi T, Qi M, Yang C, Li S, Huang K, Zheng L and Tong Q: MicroRNA-145 inhibits the growth, invasion, metastasis and angiogenesis of neuroblastoma cells through targeting hypoxia-inducible factor 2 alpha. Oncogene 33: 387-397, 2014.
23. Si ML, Zhu S, Wu H, Lu Z, Wu F and Mo YY: miR-21-mediated tumor growth. Oncogene 26: 2799-2803, 2007.

24. Cheng G: Circulating miRNAs: Roles in cancer diagnosis, prognosis and therapy. Adv Drug Deliv Rev 81: 75-93, 2014.

25. Yang Y, Gu X, Zhou M, Xiang J and Chen Z: Serum microRNAs: A new diagnostic method for colorectal cancer. Biomed Rep 1: 495-498, 2013

26. Berger F and Reiser MF: Micro-RNAs as potential new molecular biomarkers in oncology: Have they reached relevance for the clinical imaging sciences? Theranostics 3: 943-952, 2013.

27. Farazi TA, Hoell JI, Morozov P and Tuschl T: MicroRNAs in human cancer. Adv Exp Med Biol 774: 1-20, 2013.

28. Duchaine TF and Slack FJ: RNA interference and micro RNA-oriented therapy in cancer: rationales, promises, and challenges. Curr Oncol 16: 61-66, 2009.

29. Tagliaferri P, Rossi M, Di Martino MT, Amodio N, Leone E, Gulla A, Neri A and Tassone P: Promises and challenges of MicroRNA-based treatment of multiple myeloma. Curr Cancer Drug Targets 12: 838-846, 2012.

30. Orang AV and Barzegari A: MicroRNAs in colorectal cancer: from diagnosis to targeted therapy. Asian Pac J Cancer Prev 15: 6989-6999, 2014.

31. Long Z, Wang B, Tao D, Huang Y and Tao Z: Hypofractionated radiotherapy induces miR-34a expression and enhances apoptosis in human nasopharyngeal carcinoma cells. Int J Mol Med 34: 1388-1394, 2014.

32. Li G, Wang Y, Liu Y, Su Z, Liu C, Ren S, Deng T, Huang D Tian Y and Qiu Y: miR-185-3p regulates nasopharyngeal carcinoma radioresistance by targeting WNT2B in vitro. Cancer Sci 105: 1560-1568, 2014

33. Zhang C, Fang X, Li W, Shi Q, Wu L, Chen X, Huang Z, Wu P, Wang $\mathrm{Z}$ and Liao $\mathrm{Z}$ : Influence of recombinant lentiviral vector encoding miR-15a/16-1 in biological features of human nasopharyngeal carcinoma CNE-2Z cells. Cancer Biother Radiopharm 29: 422-427, 2014.

34. Li XH, Qu JQ, Yi H, Zhang PF, Yi HM, Wan XX, He QY, Ye X, Yuan L, Zhu JF, et al: Integrated analysis of differential miRNA and mRNA expression profiles in human radioresistant and radiosensitive nasopharyngeal carcinoma cells. PLoS One 9: e87767, 2014

35. Zhang JX, Qian D, Wang FW, Liao DZ, Wei JH, Tong ZT, Fu J, Huang XX, Liao YJ and Deng HX: MicroRNA-29c enhances the sensitivities of human nasopharyngeal carcinoma to cisplatin-based chemotherapy and radiotherapy. Cancer Lett 329: 91-98, 2013.

36. Qu C, Liang Z, Huang J, Zhao R, Su C, Wang S, Wang X, Zhang R, Lee $\mathrm{MH}$ and Yang H: MiR-205 determines the radioresistance of human nasopharyngeal carcinoma by directly targeting PTEN. Cell Cycle 11: 785-796, 2012.

37. Chen ZX, Sun AM, Chen Y, Liu Y, Zhan JF, Chen LH and Yuan YW: Effects of radiosensitivity and X-ray dose on miR-7 expression in nasopharyngeal carcinoma. Nan Fang Yi Ke Da Xue Xue Bao 30: 1810-1812, 2010 (In Chinese).

38. Tong L, Yuan Y and Wu S: Therapeutic microRNAs targeting the NF-kappa B signaling circuits of cancers. Adv Drug Deliv Rev 81: 1-15, 2014.

39. Zhang T, Sun Q, Liu T, Chen J, Du S, Ren C, Liao G and Yuan Y: MiR-451 increases radiosensitivity of nasopharyngeal carcinoma cells by targeting ras-related protein 14 (RAB14). Tumor Biol 35: 12593-12599, 2014.

40. Gandhi NS, Tekade RK and Chougule MB: Nanocarrier mediated delivery of siRNA/miRNA in combination with chemotherapeutic agents for cancer therapy: current progress and advances. J Control Release 194: 238-256, 2014.

41. Delibrias CC, Floettmann JE, Rowe M and Fearon DT: Downregulated expression of SHP-1 in Burkitt lymphomas and germinal center B lymphocytes. J Exp Med 186: 1575-1583, 1997.

42. Oka T, Yoshino T, Hayashi K, Ohara N, Nakanishi T, Yamaai Y, Hiraki A, Sogawa CA, Kondo E, Teramoto N, et al: Reduction of hematopoietic cell-specific tyrosine phosphatase SHP-1 gene expression in natural killer cell lymphoma and various types of lymphomas/leukemias: combination analysis with cDNA expression array and tissue microarray. Am J Pathol 159: 1495-1505, 2001

43. Sato K, Horiuchi M, Yo R and Nakarai I: A long survival case of small cell lung cancer synchronized with renal cancer. Kyobu Geka 44: 251-253, 1991 (In Japanese).

44. Amin HM,Hoshino K, Yang H,Lin Q, Lai R and Garcia-Manero G: Decreased expression level of $\mathrm{SH} 2$ domain-containing protein tyrosine phosphatase-1 (SHP-1) is associated with progression of chronic myeloid leukaemia. J Pathol 212: 402-410, 2007.

45. López-Ruiz P, Rodriguez-Ubreva J, Cariaga AE, Cortes MA and Colás B: SHP-1 in cell-cycle regulation. Anticancer Agents Med Chem 11: 89-98, 2011. 